\title{
Multidimensionality in Fluidized Nanopowder Agglomerates
}

\author{
Lilian de Martín*, Wim G. Bouwman ${ }^{\dagger}$ and J. Ruud van Ommen* \\ *Delft University of Technology, Department of Chemical Engineering, Product \& Process Engineering, \\ Julianalaan 136, 2628 BL Delft, The Netherlands \\ ${ }^{\dagger}$ Delft University of Technology, Department of Radiation, Radionuclides \& Reactors, Mekelweg 15, $2629 \mathrm{JB}$ \\ Delft, The Netherlands
}

\begin{abstract}
In recent years, the interest in fluidization as a mean to process nanoparticles is strongly increasing. Due to the small size of the nanoparticles, which makes van der Waals forces predominate, they do not fluidize as single particles but as agglomerates. Various researchers using settling experiments and bed expansion measurements conclude that fluidized agglomerates are fractal structures with a single fractal dimension of 2.5. Based on microscopy results, Wang et al. "Powder Technology 124, 152-159 (2002)" propose a hierarchical structure, which seems in contradiction with the use of only one fractal dimension as a descriptor of the whole structure. Moreover, it is not clear whether the structure presented by the agglomerates ex-situ is the same during fluidization. Hence, in this work we have characterized in-situ the internal structure of fluidized agglomerates by means of spin-echo small-angle neutron scattering (SESANS). We show that the structure of the agglomerates present at least two fractal dimensions. One of them is $\sim 2.1$ and characterizes the primary strong agglomerates. The second one is $\sim 2.8$ and characterizes the larger agglomerates formed by the primary agglomerates.
\end{abstract}

Keywords: Nanoparticles, Fluidization, Structure, Fractal

PACS: 61.46.Bc; 47.61.Jd; 62.23.Eg

\section{INTRODUCTION}

Fluidization is a promising but challenging technique for processing of nanoparticles, e.g. to produce coated nanoparticles [1]. Due to van der Waals forces, dominant at the nano-scale, nanoparticles do not fluidize individually but forming agglomerates [2]. Since the type of fluidization and transport phenomena inside the agglomerates strongly depend on the agglomerate structure, a considerably effort has been made to determine the structure of fluidized nanoagglomerates [2, 3, 4]. It is commonly accepted that nanoagglomerates are fractal structures with a fractal dimension around 2.5 [5, 3, 4], a value similar to that found in diffusion limited aggregation (DLA) [6]. However, this dimension is typically obtained with settling experiments [3], which include multiple assumptions [7] and can only focus on the macroscale of the agglomerates, since the smallest scales are not visible.

The association of a unique fractal dimension to the whole agglomerate structure seems in contradiction with the -also broadly accepted- fact that the agglomerates present a hierarchical structure $[2,8]$. If the mechanism of formation of the different hierarchical levels is different, one would expect to find different fractal dimensions in each level, reflecting the mechanism that governed its formation.

In this paper we aim to clarify this contradiction measuring in-situ the structure of the fluidized agglomerate by means of spin-echo small-angle neutron scattering
(SESANS). The results revealed that the nanoagglomerates present at least two fractal dimensions. One of them is $\sim 2.1$ and characterizes the primary strong agglomerates that the particles form. The second one is $\sim 2.8$ and characterizes the secondary agglomerates formed by the primary agglomerates. The results are in line with the hierarchical levels proposed by Wang et al. [2].

\section{EXPERIMENTAL}

Degussa $\mathrm{TiO}_{2}$ P25 nanoparticles were fluidized with pure nitrogen in a $4 \times 4 \mathrm{~cm}$ square column made of quartz and equipped with a porous plate distributor. The average particle size and density of P25 is $21 \mathrm{~nm}$ and $4000 \mathrm{~kg} / \mathrm{m}^{3}$ respectively. The bulk density was provided by the manufacturer and is $130 \pm 10 \mathrm{~kg} / \mathrm{m}^{3}$. The average size of the fluidized agglomerates was determined by means of the Richardson-Zaki method [9], obtaining a value obtained of $282 \mu \mathrm{m}$, in agreement with reported values for the same powder.

During the fluidization, the agglomerate structure was determined by means of SESANS (Reactor Institute Delft) [10]. The outcome of SESANS is a curve giving polarization vs. spin-echo length that can be related to the autocorrelation function of the structure of the fluidized powder. SESANS can measure scales from $20 \mathrm{~nm}$ up to $20 \mu \mathrm{m}$. This makes SESANS a very powerful technique to determine structural properties of nanoagglomerates.

The wavelength of the neutron beam is $\lambda=0.209 \pm$ 
$0.001 \mathrm{~nm}$. The contrast of P25 was estimated theoretically from the scattering density of the atoms and the atomic density (=particle density), obtaining $\Delta \rho_{0}=2.46$. $10^{-6} \AA^{-2}$. The voidage of the fluidized bed was obtained from transmission measurements of the fluidized and fixed bed obtaining $\phi=0.987 \pm 0.003$.

\section{MODELING AND RESULTS}

The three typical steps when analyzing a structure with SESANS is (1) to propose a model for the autocorrelation function, (2) obtain the theoretical polarization and (3) compare the theoretical and experimental polarizations. As explained, the fluidized agglomerates have been considered so far fractals with a fractal dimension $\sim 2.5$. Under this assumption, the correlation function presented by the agglomerates would be $\gamma(r) \propto r^{D_{\mathrm{f}}-3}$, or more accurately [10]:

$$
\gamma(r)=C\left(\frac{r}{r_{p}}+1\right)^{D_{\mathrm{f}}-3} \mathrm{e}^{-r / \xi}
$$

where $C$ is a prefactor, $r_{p}$ is the size of the blocks that build up the agglomerate and $\mathrm{e}^{-r / \xi}$ is a cut-off function that accounts for the finite size of the agglomerates [11]

$$
\xi^{2}=\frac{2 R_{g}^{2}}{D_{\mathrm{f}}\left(1+D_{\mathrm{f}}\right)}
$$

where $R_{g}$ is the average gyration radius of the agglomerates, assumed to be the average radius determined experimentally $\sim 140 \mu \mathrm{m}$ (see experimental section). However, in this work we postulate that one fractal dimension is not enough to characterize the scaling presented in the whole structure of the fluidized agglomerates. As explained previously, if the agglomerates are formed in a hierarchical way, they should present different dimensions depending on which mechanism governed the formation of each scale. Moreover, if primary blocks with radius $r_{c}$ and fractal dimension $D_{\mathrm{f} 1}$ are combined to form a larger agglomerate with dimension $D_{\mathrm{f} 2}$, the autocorrelation function should present a change in the fractal dimension from $D_{\mathrm{f} 1}$ to $D_{\mathrm{f} 2}$ at $\sim r_{c}$.

Thus, we propose the following model for this structure:

$$
\begin{array}{ll}
\gamma_{1}(r)=C\left(r / r_{p}+1\right)^{D_{\mathrm{f} 1}-3} & \text { for } r<r_{c} \\
\gamma_{2}(r)=C(r / a+1)^{D_{\mathrm{f} 2}-3} \exp (-r / \xi) & \text { for } r>r_{c}
\end{array}
$$

where $a$ is obtained making $\gamma_{1}(r)=\gamma_{2}(r)$ at $r=r_{c}$

$$
a=\frac{r_{c}}{\exp \left[\left(\frac{D_{\mathrm{f} 1}-3}{D_{\mathrm{f} 2}-3}\right) \ln \left(r_{c} / r_{p}+1\right)+r_{c} / \xi\right]-1}
$$

$$
\xi^{2}=\frac{2 R_{g}^{2}}{D_{\mathrm{f} 2}\left(1+D_{\mathrm{f} 2}\right)}
$$

To validate the proposed model we have simulated an agglomerate with two fractal dimensions. To do so, first we have created a cluster-cluster DLA with 2000 particles where the particle radius is one (Fig. 1a). The fractal correlation dimension of this agglomerate was 1.8. Next, we have created a particle-cluster DLA with 2000 particles and dimension 2.3. This fractal dimension is lower than the theoretical value 2.5 due to the small size of the simulated agglomerates, which gives a fractal dimension smaller than the asymptotic value [12]. The size of this particle-cluster DLA agglomerate was reduced to have a gyration radius of one and the particles of the clustercluster agglomerate were substituted by a randomly rotated version of this agglomerate. The particle size of the final agglomerate is 0.0353 .
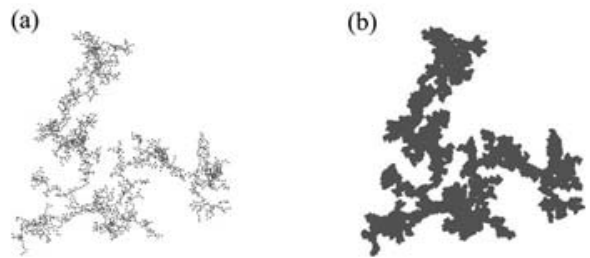

FIGURE 1. (a) Agglomerate formed by cluster-cluster DLA. $N_{p}=2000, r_{p}=1$. $D_{\mathrm{f}}=1.8$. (b) Agglomerate obtained after substituting each particle in the agglomerate (a) by an agglomerate particle-particle DLA with $N_{p}=2000$ and $D_{\mathrm{f}}=2.3$. For the agglomerate shown in (b) $N_{p}=4 \cdot 10^{6}, R_{g}=51.5, r_{a}=1$ and $r_{p}=0.0353$.

The correlation function of this agglomerate and the fitting to the bifractal model are shown in Fig. 2 and in Table 1. The agreement between the data and the model is excellent. The model predicts that primary fractal blocks with a radius 0.8 and dimension 2.3 link to form a larger fractal with dimension 1.9 , which are indeed the characteristics of the designed agglomerate. It can be concluded that when $r_{p}$ and $R_{g}$ are provided and the proposed model is applied over a two level structure, it returns the radius $r_{c}$ and dimension $D_{\mathrm{f} 1}$ of the primary fractal blocks and the dimension $D_{\mathrm{f} 2}$ of the secondary fractal.

TABLE 1. Comparison between the fractal dimensions and the critical length of the cluster shown in Fig. 1b and the parameters obtained from the model.

\begin{tabular}{lrrr}
\hline & $D_{\mathrm{f} 1}$ & $D_{\mathrm{f} 2}$ & $r_{c}$ \\
\hline Original parameters & 2.3 & 1.8 & 1 \\
Retrieved parameters & 2.3 & 1.9 & 0.8 \\
\hline
\end{tabular}




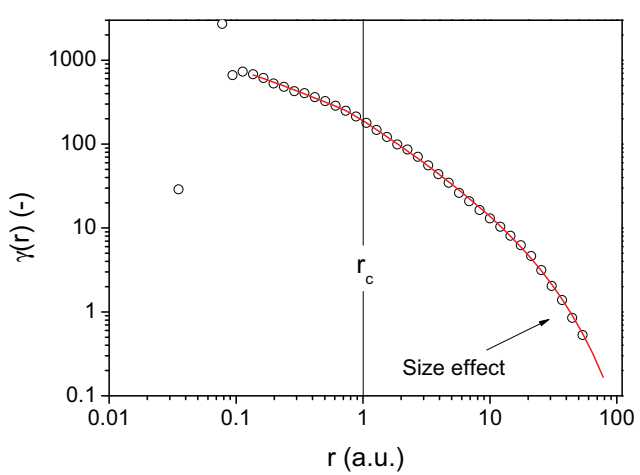

FIGURE 2. Fitting of the bifractal model to the correlation function of the agglomerate shown in Fig. $1 \mathrm{~b}$.

The next step is to calculate the theoretical polarization provided by each model [10]. It is important to remark that this procedure works with the normalized correlation function, what means that the prefactor $C$ is 1 in both models. Additionally, the following equations relate the correlation function in the bifractal model to the polarization. For the monofractal model the approach is equivalent but the integrals are not split. The correlation length $\Gamma$ is defined as

$$
\Gamma=2 \int_{0}^{r_{c}} \gamma_{1}(r) \mathrm{d} r+2 \int_{r_{c}}^{\infty} \gamma_{2}(r) \mathrm{d} r
$$

The Abel projection for $z<z_{c}$ is

$$
G(z)=\frac{2}{\Gamma}\left(\int_{z}^{z_{c}} \frac{\gamma_{1}(r) r}{\sqrt{r^{2}-z^{2}}} \mathrm{~d} r+\int_{z_{c}}^{\infty} \frac{\gamma_{2}(r) r}{\sqrt{r^{2}-z^{2}}} \mathrm{~d} r\right),
$$

and for $z>z_{c}$ is

$$
G(z)=\frac{2}{\Gamma} \int_{z}^{\infty} \frac{\gamma_{2}(r) r}{\sqrt{r^{2}-z^{2}}} \mathrm{~d} r
$$

Note that $z_{c}=r_{c}$. The relationship between the polarization curve and the projection of the autocorrelation function is

$$
P_{t h}(z)=\mathrm{e}^{\Sigma_{t}(G(z)-1)}
$$

where

$$
\Sigma_{t}=\lambda^{2} t\left(\Delta \rho_{0}\right)^{2} \phi(1-\phi) \Gamma
$$

$\lambda$ is the wavelength of the neutron beam, $t$ is the thickness of the sample $(40 \mathrm{~mm}), \Delta \rho_{0}$ is the contrast and $\phi$ is the voidage (see experimental section). Once $\gamma(r)$ and $P_{t h}$ are related, it is possible to write a routine to find the parameters of $\gamma(r)$ that minimize the least square error between $P_{\text {exp }}$ and $P_{t h}$.
The SESANS measurements and the theoretical polarization given by the monofractal model are shown in Fig. 3. The black dashed line with $D_{\mathrm{f}}=2.36$ represents the best fitting for the experimentally obtained $\phi$. The fitting is very poor, indicating that the model does not capture properly the structural properties of the agglomerates. To further demonstrate that the monofractal approach does not characterize the scaling in the whole range of scales we have repeated the fitting, but this time leaving $\phi$ as a fitting parameter, together with $D_{\mathrm{f}}$. In this way, it is possible to see the best performance of the model. The fitting is plotted with a gray line, obtaining a fractal dimension of 2.80 and an unrealistic voidage value of 0.997 . Even in the most favorable scenario the monofractal model is clearly biased, overestimating the polarization at the smaller scales.

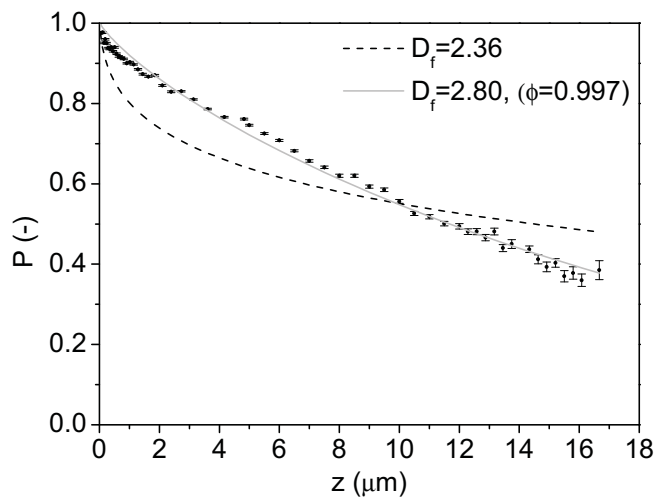

FIGURE 3. Polarization curve predicted with the conventional monofractal model. The black dashed line represents the best fitting for the experimentally determined $\phi=0.987$. The gray line represents the best possible fitting for the monofractal model, since $\phi$ has been left as fitting parameter, obtaining $\phi=0.997$.

The fitting obtained with the bifractal model is shown in Fig. 4. To determine the influence of the error in $\lambda$, $t$ and $\phi$ on the fitting parameters, we have assumed that each experimental variable follows a Gaussian distribution with its confidence interval equal to $2 \sigma$. Then, we have done 1000 fittings varying randomly the values of the experimental variables according to their Gaussian probability. The fitting parameters and the confidence intervals are shown in Table 2.

TABLE 2. Fitting parameters and confidence intervals for the mono and bifractal model. The confidence intervals are $2 \sigma$.

\begin{tabular}{lrrr}
\hline Model & $D_{\mathrm{f} 1}$ & $D_{\mathrm{f} 2}$ & $r_{c}(\mu \mathrm{m})$ \\
\hline Monofractal & $2.35 \pm 0.03$ & - & - \\
Bifractal & $2.1 \pm 0.2$ & $2.85 \pm 0.01$ & $0.13 \pm 0.05$ \\
\hline
\end{tabular}




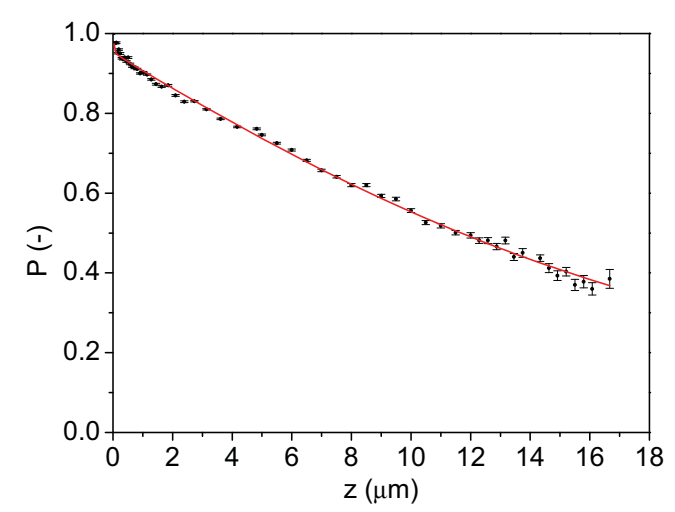

FIGURE 4. Polarization curve predicted with the bifractal model. The fitting parameters are shown in Table 2.

Contrary to the monofractal model (Fig. 3), the bifractal characterizes the structural properties in the whole range of sizes studied (Fig. 4). One could argue that the better fitting is simply due to the two extra fitting parameters of this model respect to the monofractal approach. However, a thorough analysis of the values obtained for $D_{\mathrm{f} 1}, D_{\mathrm{f} 2}$ and $r_{c}$ reveals the physical meaning. Following the same reasoning that carried out in the validation of the model, the interpretation of the results is that primary building blocks of $\sim 260 \mathrm{~nm}$ and dimension $\sim 2.1$ build up larger agglomerates with dimension $\sim 2$.8. Wang et al. [2] claim the nanoparticles first link to form primary open agglomerates with a size of a few hundred of $\mathrm{nm}$ and then, these primary agglomerates form more compact secondary agglomerates. Additionally, a studied carried out by Teleki et al. [13] concluded that titania P25 first form strong aggregates with a size $\sim 200 \mathrm{~nm}$ and they form larger agglomerates afterward. The dimension $\sim 2.1$ that the model determines at the smallest scales corresponds to the open strong primary aggregates that P25 nanoparticles form in first instance, probably in the manufacturing process. The dimension $\sim 2.8$ at largest scales corresponds to the secondary agglomerates formed by the primary agglomerates.

One remarkable aspect of these dimensions is that they do not represent the conventional particle-cluster $\left(D_{\mathrm{f}}=2.5\right)$ and cluster-cluster $\left(D_{\mathrm{f}}=1.8\right)$ DLA mechanisms. This makes sense as long as these mechanisms do not take into account breakage of the agglomerates and rearrangement of the nanoparticles due to compression or sintering processes [14, 15]. Hyeon-Lee et al. [16] showed that for titania aggregates synthesized in a diffusion flame reactor the fractal dimension ranges from 1.6 to 2.5, which is in agreement with the value found in this work for the primary agglomerates.

\section{CONCLUSIONS}

We have proven the multidimensionality of the fluidized nanopowder agglomerates and validated their hierarchical structure, first proposed by [2]. The fractal dimension changes from one to other hierarchical level as a result of the different mechanisms that govern their formation. This is opposite to the classical description that uses only one fractal dimension to characterize the scaling in the whole structure. The fractal dimensions found can help to unravel the mechanisms that form each hierarchical level and to explain diffusion processes inside the agglomerates.

\section{ACKNOWLEDGEMENTS}

We acknowledge Chris Duif for performing the SESANS measurements.

\section{REFERENCES}

1. J. van Ommen, J. Valverde, and R. Pfeffer, Journal of Nanoparticle Research 14, 1-29 (2012).

2. Y. Wang, G. Gu, F. Wei, and J. Wu, Powder Technology 124, 152-159 (2002).

3. A. Castellanos, J. M. Valverde, and M. A. S. Quintanilla, Phys. Rev. E 64, 041304 (2001).

4. J. Quevedo, R. Pfeffer, Y. Shen, R. Dave, H. Nakamura, and S. Watano, AIChE Journal 52, 2401-2412 (2006), ISSN 1547-5905.

5. C. H. Nam, R. Pfeffer, R. N. Dave, and S. Sundaresan, AIChE Journal 50, 1776-1785 (2004).

6. T. A. Witten, and L. M. Sander, Physical Review Letters 47, 1400-1403 (1981).

7. P. Tang, and J. Raper, Powder Technology 123, 114-125 (2002).

8. J. M. Valverde, and A. Castellanos, AIChE Journal 52, 838-842 (2006).

9. C. Zhu, Q. Yu, R. N. Dave, and R. Pfeffer, AIChE Journal 51, 426-439 (2005).

10. R. Andersson, L. F. van Heijkamp, I. M. de Schepper, and W. G. Bouwman, Journal of Applied Crystallography 41, 868-885 (2008).

11. J. Teixeira, Journal of Applied Crystallography 21, 781-785 (1988).

12. P. Meakin, Journal of Sol-Gel Science and Technology 15, 97-117 (1999).

13. A. Teleki, R. Wengeler, L. Wengeler, H. Nirschl, and S. Pratsinis, Powder Technology 181, 292 - 300 (2008).

14. J. H. Scheckman, P. H. McMurry, and S. E. Pratsinis, Langmuir 25, 8248-8254 (2009).

15. M. L. Eggersdorfer, D. Kadau, H. J. Herrmann, and S. E. Pratsinis, Langmuir 27, 6358-6367 (2011).

16. J. Hyeon-Lee, G. Beaucage, S. E. Pratsinis, and S. Vemury, Langmuir 14, 5751-5756 (1998). 